\title{
Image representation and compression with the fractional Fourier transform
}

\author{
I. Şamil Yetik ${ }^{\text {a,* }}$, M. Alper Kutay ${ }^{b}$, Haldun M. Ozaktas ${ }^{\text {c }}$ \\ ${ }^{a}$ Department of Electrical Engineering and Computer Science, University of Illinois at Chicago, Chicago, IL 60607, USA \\ b TUBITAK-UEKAE, No. 221, Kat 15, TR-06100 Kavaklidere, Ankara, Turkey \\ c Department of Electrical Engineering, Bilkent University, TR-06533 Bilkent, Ankara, Turkey \\ Received 8 March 2001; received in revised form 25 July 2001; accepted 25 July 2001
}

\begin{abstract}
We discuss the application of fractional Fourier transform-based filtering configurations to image representation and compression. An image can be approximately represented (and stored or transmitted) as the coefficients of the minimum mean square filtering configuration approximating the image matrix. An order of magnitude compression is possible with moderate errors with the raw method. While inferior to commonly available compression algorithms, the results presented correspond to the basic method without any refinement or combination with other techniques, suggesting that the approach may hold promise for future development. Regardless of its practical usefulness, the fact that the information inherent in an image can be decomposed or factored into fractional Fourier domains is of considerable conceptual significance. The information contained in the image is distributed to the different domains in an unequal way, making some domains more dispensible than others in representing the image. () 2001 Published by Elsevier Science B.V.
\end{abstract}

There has been a tremendous amount of work on data compression in general and image compression in particular, leading to efficient compression algorithms. In this paper we discuss a novel way of representing images based on fractional Fourier-domain filtering configurations [1, 2], leading to a method for compressing images.

Space- and frequency-domain filtering are special cases of fractional Fourier-domain filtering (Fig. 1(a)-(c)) [3,4]. Fractional Fourier-domain

\footnotetext{
${ }^{*}$ Corresponding author. Fax: +1-312-413-0024.

E-mail address: syetik1@uic.edu (i.ş. Yetik).
}

filtering consists of (i) taking the fractional Fourier transform of the input signal, (ii) multiplication with a filter function, and (iii) taking the inverse fractional Fourier transform of the result. The fractional version of the optimal Wiener filtering problem has been studied in detail in Ref. [4]. Fractional Fourier-domain filtering has been further generalized to multi-stage and multi-channel filtering (Fig. 1(e) and (f)). In multi-stage filtering $[2,5]$ the input is first transformed into the $a_{1}$ th domain, where it is multiplied by a filter $h_{1}$. The result is then transformed back into the original domain. This process is repeated $M$ times. Denoting the diagonal matrix corresponding to multiplication by the $k$ th filter by $\boldsymbol{\Lambda}_{k}$, we can write 


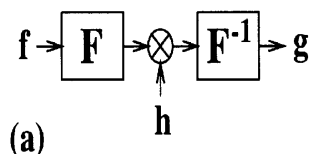

(a)

(b)<smiles>[V]=[Tl]</smiles>

(d)

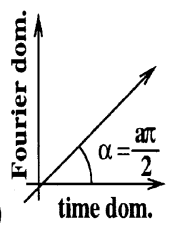

(c)

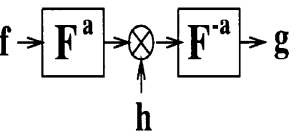

c)

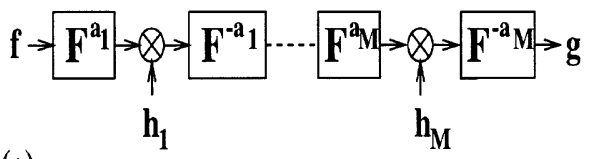

(e)

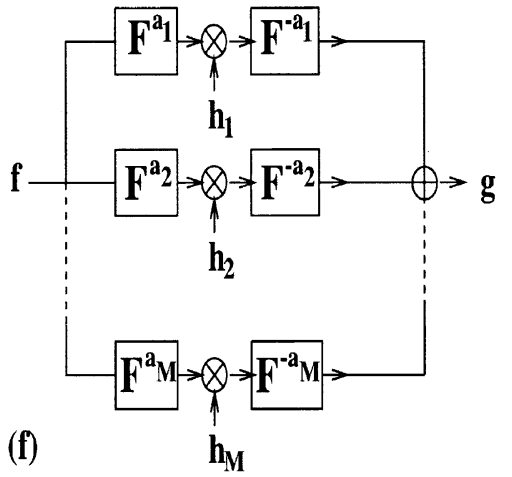

Fig. 1. (a) Fourier-domain filtering, (b) space-domain filtering, (c) $a$ th order fractional Fourier-domain filtering, (d) fractional Fourier domains, (e) multi-stage filtering, (f) multi-channel filtering.

the following expression for the overall effect of the multi-stage filtering configuration:

$\mathbf{T}_{\mathrm{ms}}=\left[\mathbf{F}^{-a_{M}} \boldsymbol{\Lambda}_{h_{M}} \ldots \mathbf{F}^{a_{2}-a_{1}} \boldsymbol{\Lambda}_{h_{1}} \mathbf{F}^{a_{1}}\right]$,

where $\mathbf{T}_{\mathrm{ms}}$ is a matrix representing the overall multi-stage filtering configuration and $\mathbf{F}^{a_{k}}$ denotes the discrete fractional Fourier transform matrix [6]. Multi-channel filtering circuits [2,7] consist of $M$ single-stage blocks in parallel. For each channel $k$, the input is transformed to the $a_{k}$ th domain, multiplied by a filter $h_{k}$ and then transformed back. Now we can write the following expression for the overall effect of the multi-channel filtering configuration:
$\mathbf{T}_{\mathrm{mc}}=\left[\sum_{k=1}^{M} \mathbf{F}^{-a_{k}} \boldsymbol{\Lambda}_{k} \mathbf{F}^{a_{k}}\right]$,

where $\mathbf{T}_{\mathrm{mc}}$ is a matrix representing the overall multi-channel filtering configuration. It is possible to further generalize these filtering configurations by using parallel and series arrangements together; such systems have been called generalized filtering circuits [1,2].

Fractional Fourier transform has found many applications in optics in general [8] and fractional Fourier transform-based filtering circuits have found applications in optical and digital signal and image restoration, signal and system synthesis, synthesis of mutual intensity distributions, and fast implementation of shift-variant linear systems $[1,2,5,7]$.

In multi-stage and multi-channel filtering configurations, there are two categories of unknowns, the fractional Fourier transform orders and the filter coefficients. The problem of finding the optimal filter coefficients, given the transform orders has been solved in Refs. [2,4,5] using a minimum mean square error approach. On the other hand, the problem of optimizing multiple orders has not yet been addressed, and in most cases the orders have been chosen uniformly. In this paper we have attempted to optimize over the orders for multichannel filtering by first finding the optimal filter coefficients for a larger number of uniformly chosen orders and then maintaining the most important ones.

In Refs. [2,5,7] fractional Fourier transformbased filtering configurations have been used for synthesizing linear space-variant systems, represented by some matrix $\mathbf{T}$. It was shown that for many such systems encountered in various applications, it is possible to approximate the system with a multi-stage or multi-channel configuration with acceptable mean square error, by using a small or moderate number $(M)$ of stages or channels. Since the cost of implementing the fractional Fourier transform (optically or digitally) is similar to the cost of implementing the ordinary Fourier transform, this leads to a fast implementation of the space-variant system in question. For instance, for digital systems, the cost becomes $\mathrm{O}(M N \log N)$, 
which should be compared to the cost $\mathrm{O}\left(N^{2}\right)$ for direct implementation of linear systems.

In this paper we interpret the matrix $\mathbf{T}$ not as representing a linear system, but as representing a two-dimensional signal or image. Thus the filtering coefficients in the multi-stage or multi-channel approximation of this matrix, can be used to approximately represent and reconstruct this matrix and the associated image. In other words, the optimal filtering coefficients minimizing the mean square error between the original matrix and its multi-stage and multi-channel approximation, are taken as the compressed version of the image. The cited work on synthesis of space-variant systems for fast implementation shows that satisfactory approximations are possible with moderate numbers of filters and hence large reductions in implementation cost. Therefore, it seems worth investigating whether similar approximations with similar reductions in cost (measured by the compression ratio) is possible when these configurations are used for image compression. Since the original image has $N^{2}$ pixels and the compressed data has $N M$ pixels, the compression ratio is $N / M$.

In the multi-channel case it is possible to analytically find the optimal filter coefficients, provided the transform orders have been chosen. In practice, however, an iterative method is preferred. In the multi-stage case it is not possible to find analytic solutions, so an iterative method must be used to begin with. The criterion of optimality in approximating $\mathbf{T}$ with $\mathbf{T}_{\mathrm{mc}}$ or $\mathbf{T}_{\mathrm{ms}}$ is minimum mean square distance.

In the multi-channel filtering case, we have also considered the improvement of optimizing over the orders by first finding the optimal filter coefficients for a larger number of uniformly chosen orders and then maintaining the most important ones. More specifically, we start with several times the number of orders $M$ we are eventually going to use. Then, the $M$ orders resulting in filters with the highest energy are chosen, and the other branches of the multi-channel configuration are eliminated. Finally, with the orders thus chosen, we re-optimize the filter coefficients as before.

The compression method proposed is tested on the $128 \times 128$ image shown in Fig. 2(a). Fig. 2(b) shows the trade-off between the reconstruction (a)

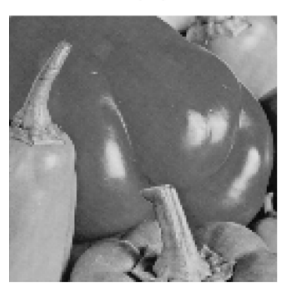

(c)

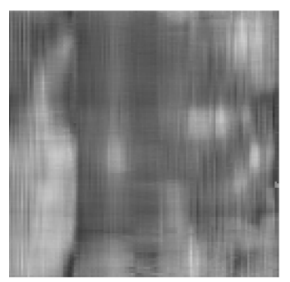

(e)

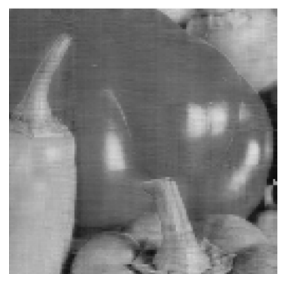

(b)

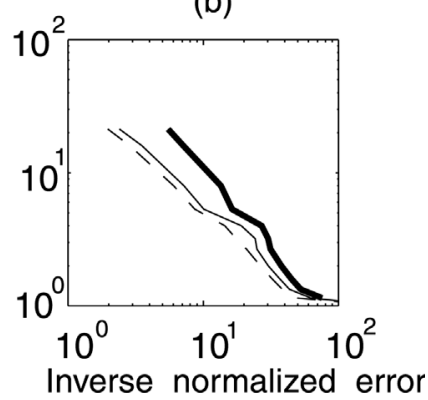

(d)

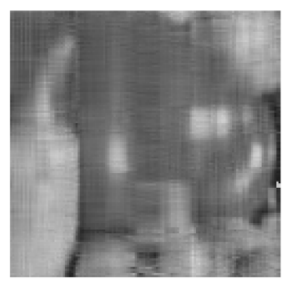

(f)

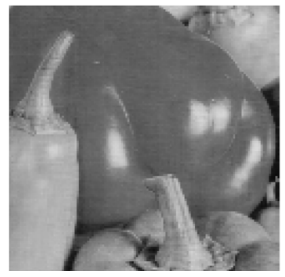

Fig. 2. (a) Original image, (b) compression ratio vs inverse normalized error: multi-channel (dashed line), multi-stage (solid line), multi-channel with optimized orders (bold line). (c) Reconstructed images with compression ratio 32, (d) 21.3, (e) 8, (f) 5.3. Parts (c) and (d) represent too much error to be considered compressed versions of the original image. However, they have been shown to illustrate the dependence of the error on the number of coefficients used.

error and compression ratio. The mean square error has been normalized by the energy of the original image. The horizontal axis of the plot is the inverse of this normalized error. We see that the multi-channel and multi-stage configurations give comparable results, though the multi-stage configuration is slightly better. Optimizing over the orders for the multi-channel case results in tangible improvements.

Fig. 2(c)-(f) shows illustrative results obtained with the multi-stage configuration. Although the 
order-optimized multi-channel case yields smaller errors, we present results for the multi-stage configuration so as to illustrate the performance of the method in its rawest, most basic form. Whereas we observe that nearly an order of magnitude compression is possible with moderate errors, larger compression ratios are accompanied by larger errors.

Unfortunately, we observe that the use of fractional Fourier-domain filtering configurations for image compression, does not yield results as good as those obtained when they are used for synthesis and fast implementation of shift-variant linear systems. In its present form, the proposed idea does not yield better results than presently available compression algorithms. However, we emphasize that the results presented reflect the performance of the basic method in its rawest and barest form; we merely represent the image with the filter coefficients which make the forms given in Eqs. (1) and (2) as close as possible to the image matrix. Further refinement and development of the method and its combination and joint use with other techniques may lead to full-fledged compression algorithms with better performance. (One way of generalizing the method, which can lead to potentially higher compression ratios with similar errors is to employ filtering circuits based on linear canonical transforms, rather than fractional Fourier transforms [9].)

Moreover, regardless of the performance that can ultimately be obtained with improvements of the present idea, the fact that the information inherent in an image can be decomposed or factored into fractional Fourier domains in the manner described is of considerable conceptual significance. In a sense, these domains "span" a certain space which is a subset of the image space, although the precise nature of this is difficult to ascertain in the nonlinear multi-stage case. The information contained in the image is distributed to the different domains in an unequal way, making some domains more dispensible than others in representing the image. Exploring and exploiting these issues seem potentially rewarding.

\section{References}

[1] H.M. Ozaktas, M.A. Kutay, D. Mendlovic, Introduction to the fractional Fourier transform and its applications, in: P.W. Hawkes (Ed.), Advances in Imaging and Electron Physics, vol. 106, Academic Press, San Diego, CA, 1999, pp. 239-291 (Chapter 4);

H.M. Ozaktas, Z. Zalevsky, M.A. Kutay, The Fractional Fourier Transform with Applications in Optics and Signal Processing, Wiley, New York, 2001.

[2] M.A. Kutay, M.F. Erden, H.M. Ozaktas, O. Arıkan, Ö. Güleryüz, Ç. Candan, Opt. Lett. 23 (1998) 1069-1071; M.A. Kutay, M.F. Erden, H.M. Ozaktas, O. Arıkan, Ç. Candan, Ö. Güleryüz, Proceedings of the 1998 IEEE International Conference on Acoustics, Speech, and Signal Processing, IEEE, Piscataway, NJ, 1998, pp. 3433-3436; M.A. Kutay, H. Özaktaş, M.F. Erden, H.M. Ozaktas, O. Arıkan, Proceedings of the 1998 IEEE-SP International Symposium on Time-Frequency and Time-Scale Analysis, IEEE, Piscataway, NJ, 1998, pp. 481-484.

[3] H.M. Ozaktas, B. Barshan, D. Mendlovic, L. Onural, J. Opt. Soc. Am. A 11 (1994) 547-559;

H.M. Ozaktas, B. Barshan, D. Mendlovic, Opt. Rev. 1 (1994) 15-16.

[4] M.A. Kutay, H.M. Ozaktas, O. Arıkan, L. Onural, IEEE Trans. Signal Process. 45 (1997) 1129-1143.

[5] M.F. Erden, M.A. Kutay, H.M. Ozaktas, IEEE Trans. Signal Process. 47 (1999) 1458-1462;

M.F. Erden, H.M. Ozaktas, J. Opt. Soc. Am. A 15 (1998) 1647-1657.

[6] Ç. Candan, M.A. Kutay, H.M. Ozaktas, IEEE Trans. Signal Process. 48 (2000) 1329-1337.

[7] M.A. Kutay, H. Özaktaş, H.M. Ozaktas, O. Arıkan, Signal Process. 77 (1999) 105-109;

İ.Ş. Yetik, M.A. Kutay, H. Özaktaş, H.M. Ozaktas, Proceedings of the 2000 IEEE International Conference on Acoustics, Speech, and Signal Processing, IEEE, Piscataway, NJ, 2000, pp. I:93-96.

[8] H.M. Ozaktas, D. Mendlovic, Opt. Commun. 101 (1993) 163-169;

D. Mendlovic, H.M. Ozaktas, J. Opt. Soc. Am. A 10 (1993) 1875-1881;

T. Alieva, J. Opt. Soc. Am. A 13 (1996) 1189-1192;

L.M. Bernardo, O.D.D. Soares, J. Opt. Soc. Am. A 11 (1994) 2622-2626;

A.W. Lohmann, D. Mendlovic, Z. Zalevsky, Fractional transformations in optics, Progress in Optics XXXVIII, Elsevier, Amsterdam, 1998, pp. 263-342 (Chapter IV);

H.M. Ozaktas, D. Mendlovic, J. Opt. Soc. Am. A 12 (1995) 743-751;

H.M. Ozaktas, M.F. Erden, Opt. Commun. 143 (1997) 7586.

[9] B. Barshan, M.A. Kutay, H.M. Ozaktas, Opt. Commun. 135 (1997) 32-36. 